\title{
A case of Trousseau's syndrome due to intrahepatic cholangiocarcinoma with an extremely high level of CA19-9
}

\author{
Ryosuke Sasaki, Yuki Ohya ${ }^{1 *}$, Shintaro Hayashida', Yuto Maeda', Shuei Murahashi², Sayahito Kumamoto', \\ Akira Tsuji ${ }^{1}$, Hidekatsu Shibata ${ }^{1}$, Kunitaka Kuramoto ${ }^{1}$, Hironori Hayashi ${ }^{1}$, Kazumi Kuriwaki ${ }^{3}$, Masayoshi lizaka ${ }^{1}$, \\ Osamu Nakahara ${ }^{1}$ and Yukihiro Inomata ${ }^{1}$
}

\begin{abstract}
Background: Trousseau's syndrome is a cancer-associated thrombosis. Trousseau's syndrome with cholangiocarcinoma is a rare condition with poor prognosis.

Case presentation: A 59-year-old female was admitted to our hospital with abdominal pain, headache, and nausea. Abdominal enhanced computed tomography revealed liver tumor, splenic infarction, and bilateral renal infarction. Multiple acute cerebral infarctions were also detected by magnetic resonance imaging. Her preoperative serum levels of carbohydrate antigen 19-9 (CA19-9) and carcinoembryonic antigen (CEA) were $>120,000 \mathrm{U} / \mathrm{mL}$ and $589.6 \mathrm{ng} / \mathrm{mL}$, respectively, which were extremely high. Histopathology after right hepatectomy revealed moderately differentiated adenocarcinoma consistent with intrahepatic cholangiocarcinoma. Her serum levels of CA19-9 were trending down to 9029.2 and $2659.8 \mathrm{U} / \mathrm{mL}$ at 1 and 3 weeks after surgery, respectively. However, at 7 weeks after surgery, her CA19-9 levels increased in the presence of positive imaging findings in the remnant liver, hilar lymph nodes, and peritoneal cavity. The initiation of combination chemotherapy including gemcitabine and cisplatin had a significant effect. The patient was doing well at 6 months after the surgery.
\end{abstract}

Conclusion: This rare case of Trousseau's syndrome due to cholangiocarcinoma suggests that extremely high CA19-9 levels might be a pathogenic factor of this syndrome.

Keywords: Trousseau's syndrome, Intrahepatic cholangiocarcinoma, Carbohydrate antigen 19-9

\section{Background}

Armand Trousseau was the first to describe the association of thrombotic episodes with an underlying malignancy [1-5]. Recent definitions restrict Trousseau's syndrome to unexplained thrombotic events that occur before or concomitantly with the presentation of visceral malignancies [3]. The prognosis of Trousseau's syndrome, which is often diagnosed in patients with terminal cancer, is extremely poor. While the precise mechanism underlying Trousseau's syndrome remains

\footnotetext{
* Correspondence: pedsurg-oya@kumamotoh.johas.go.jp

'Department of Surgery, Kumamoto Rosai Hospital, 1670 Takehara-machi,

Yatsushiro, Kumamoto 866-8533, Japan

Full list of author information is available at the end of the article
}

unknown, hypercoagulability might be initiated by mucins produced by an adenocarcinoma [4]. Intriguingly, Trousseau's syndrome is rarely reported in patients with cholangiocarcinoma [3]. We herein report the case of a patient with intrahepatic cholangiocarcinoma who presented with Trousseau's syndrome manifesting as thromboembolic lesions in multiple organs including the brain and was treated with right hepatectomy followed by systemic chemotherapy.

\section{Case presentation}

A 59-year-old female visited an emergency room with the chief complaints of abdominal pain and dizziness and was diagnosed with gastroenteritis and vertigo.

\section{Springer Open}

(c) The Author(s). 2020 Open Access This article is licensed under a Creative Commons Attribution 4.0 International License, which permits use, sharing, adaptation, distribution and reproduction in any medium or format, as long as you give appropriate credit to the original author(s) and the source, provide a link to the Creative Commons licence, and indicate if changes were made. The images or other third party material in this article are included in the article's Creative Commons licence, unless indicated otherwise in a credit line to the material. If material is not included in the article's Creative Commons licence and your intended use is not permitted by statutory regulation or exceeds the permitted use, you will need to obtain permission directly from the copyright holder. To view a copy of this licence, visit http://creativecommons.org/licenses/by/4.0/. 
Three days later, she was readmitted to the emergency room because of worsening abdominal pain and difficulty in movement due to headache and nausea. Plain whole-body computed tomography (CT) showed suspicious cerebral infarction and a low-density mass lesion in right liver lobe. Subsequent head magnetic resonance imaging (MRI) confirmed infarcted areas in multiple blood vessels defined as an embolic shower. Contrastenhanced CT revealed about $9 \times 6 \times 4 \mathrm{~cm}$-sized and illdefined mass with several daughter nodules in the posterior segment of the right hepatic lobe, splenic infarction, and bilateral renal infarctions (Fig. 1). The liver hypodense mass had a peripheral rim-like enhancement during the arterial phase and contacted with diaphragm, retroperitoneum, and inferior vena cava. The tumor in the liver was diagnosed as intrahepatic mass-forming cholangiocarcinoma. There was no suspicion of other gastrointestinal or gynecological malignancies. At that time of diagnosis, the level of carbohydrate antigen 19-9 (CA19-9) was > 120,000 U/mL, above the detectable range, and the level of carcinoembryonic antigen (CEA) was $211.7 \mathrm{ng} / \mathrm{mL}$. The coagulation profile of the patient was as follows: platelet count, $8.7 \times 10^{4} / \mu \mathrm{L}$; D-dimer, $36.6 \mu \mathrm{g} / \mathrm{mL}$; prothrombin time-international normalized ratio, 1.16; and activated partial thromboplastin time, $28.1 \mathrm{~s}$. Ultrasonography of the carotid arteries and lower limbs, transthoracic echocardiography, and Holter electrocardiogram did not reveal the source of embolism. Therefore, the patient was diagnosed with Trousseau's syndrome due to intrahepatic cholangiocarcinoma.

Continuous intravenous infusion of unfractionated heparin at a dose of 10,000 U per day was started immediately. However, she developed left hemiplegia 1 week later, and head MRI revealed thrombosis of the right middle cerebral artery. Activated partial thromboplastin time value was $23.4 \mathrm{~s}$ at that time in spite of continuous administration of heparin. She was transferred to a hospital where endovascular treatment was possible, and thrombectomy was performed. Although left paralysis remained after thrombectomy, she returned to our hospital for the treatment of intrahepatic cholangiocarcinoma. In the meantime, she was treated with continuous infusion of unfractionated heparin at a dose of 15,000 U per day. After the thrombectomy, activated partial thromboplastin time value was kept nearly $40.0 \mathrm{~s}$ with administration of heparin.

Preoperative examination did not show any issues with cardiopulmonary function. Abdominal enhanced CT and MRI showed a tumor in the posterior segment of the right hepatic lobe (Fig. 1). Hepatic reserve after right hepatectomy was confirmed with indocyanine green clearance test and technetium-99m diethylenetriamine penta-acetic acid-galactosyl human serum albumin (99mTc-GSA) liver scintigraphy (data not shown).
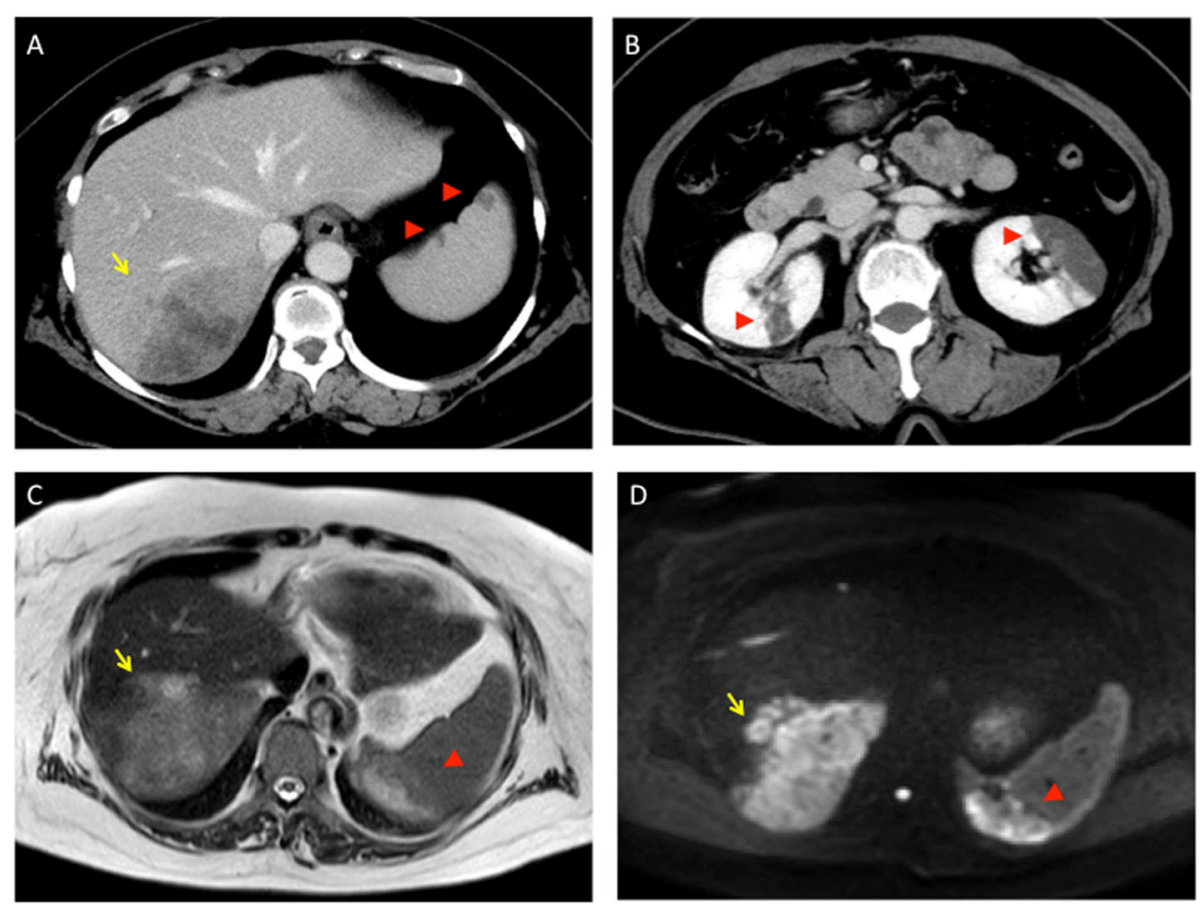

Fig. 1 Abdominal enhanced CT and MRI findings. a CT showing a tumor in the posterior segment of the right hepatic lobe (arrow) and splenic infarctions (arrowhead). b CT showing renal infarctions in both kidneys (arrowhead). c, d MRI showing a tumor in the posterior segment of the right hepatic lobe (arrow) and splenic infarctions (arrowhead). CT, computed tomography; MRl, magnetic resonance imaging 
Although the preoperative serum levels of CA19-9 and CEA were extremely high, the preoperative $\mathrm{D}$-dimer, fibrin degradation product (FDP), and fibrinogen levels were slightly higher than the normal limits (Table 1). Protein C level was $154 \%$ (normal 70-150), protein S level was 164\% (normal 65-135), and cardiolipin antibodies were normal (IgG, < 8; IgM, 5).

During the surgery, anticoagulation was performed with subcutaneous injection of low-molecular-weight heparin at a dose of $5000 \mathrm{U}$ per $12 \mathrm{~h}$. In surgery, the tumor was exposed on the liver surface and partially adhered to the diaphragm and retroperitoneum. Right hepatectomy was performed with combined resection of the invading tumor.

The resected tumor was $9 \times 6 \times 6 \mathrm{~cm}$ in size, and the pathological diagnosis was moderately differentiated intrahepatic cholangiocarcinoma (pT4pNOM0 pStage IVA, vp1, vv2, b1, UICC8th) (Fig. 2). Intraoperative irrigation cytology was negative, and lymph nodes of no.12 did not show metastasis. Tumor invasion to the resected diaphragm was pathologically confirmed, but there was no invasion to the retroperitoneum.

Immunohistochemistry was performed for cancer antigen 125 (CA125; clone M3519; Dako, Glostrup, Denmark), CEA (clone M7072; Dako), cell surfaceassociated mucin 1 recognized by the cancer antigen 15 3 (CA15-3) epitope (MUC-1; clone MA552; Leica Biosystems, Nussloch, Germany), and CA19-9 (M3517; Dako). As seen in Fig. 3, strong expression levels of CA19-9, CEA, and MUC-1 were observed in the cytoplasm and membrane of cholangiocarcinoma cells.

After the surgery, the patient's general condition was good and no neurological changes were observed. The perioperative changes in serum tumor maker levels are shown in Fig. 4. Serum CA19-9 remained above detectable levels until 4 days after surgery and decreased to 43,068 U/ $\mathrm{mL}$ on postoperative day 4 . By imaging, the splenic and renal infarcts were slightly enlarged and a new subacute subcortical hemorrhage in the right occipital lobe was observed on postoperative day 7 . Starting at 1 week after surgery, anticoagulant therapy was resumed by continuous intravenous infusion of unfractionated heparin at a dose of 15,000 $\mathrm{U}$ per day. At 1-month postoperative evaluation, no new neurological changes were observed and there were no exacerbations in the infarcts and hemorrhagic lesions. The serum levels of CA19-9 started to increase, reaching 9459.4 and $80,256.2 \mathrm{U} / \mathrm{mL}$ at 4 and 8 weeks after the surgery, respectively. Although enhanced CT did not show recurrence, positron emission tomography suggested intrahepatic recurrence, hilar lymph node metastasis, and peritoneal dissemination at 7 weeks after the surgery (Fig. 5). Therefore, the patient was initiated on combination chemotherapy with gemcitabine and cisplatin at 8 weeks after the surgery. The chemotherapy regimen comprised cisplatin $\left(25 \mathrm{mg} / \mathrm{m}^{2}\right.$ body surface area) followed by gemcitabine $\left(1000 \mathrm{mg} / \mathrm{m}^{2}\right.$ body surface area) [6], both administered on days 1 and 8 , every 3 weeks. This treatment was effective in stabilizing CA19-9 levels (Fig. 4) but did not cure the tumor recurrence.

Continuous intravenous heparin administration was changed to subcutaneous injection of low-molecularweight heparin at a dose of $5000 \mathrm{U}$ per $12 \mathrm{~h}$, and she was discharged 12 weeks after the surgery. She has been subsequently followed in our outpatient clinic without further thrombosis during the last 6 months after surgery, and there has been no tumor progression.

\section{Discussion}

Tumors associated with Trousseau's syndrome are usually mucin-producing adenocarcinomas derived from visceral organs. However, few studies have reported an association between cholangiocarcinoma and thromboembolism $[3,7,8]$. The prognosis is extremely poor in patients with Trousseau's syndrome related to primary cholangiocarcinoma $[3,7,8]$, which was first reported by Ching in 1991 [7]. Eleven cases of Trousseau's syndrome developed in intrahepatic cholangiocarcinoma have been reported in English [3, 7, 8]. Only three patients of eleven survived for more than 3 months, and there was no patient who underwent liver resection. However, the

Table 1 Tumor maker levels and coagulation tests

\begin{tabular}{lll}
\hline [normal range] & Pre-operative TMs and CTs & TMs and CTs at POW4 \\
\hline CA19-9 (U/mL) [0.0-37.0] & $>12,0000$ & 4905.5 \\
CEA (ng/mL) [0.0-5.0] & 589.6 & 6.3 \\
CA125 (U/mL) [0.0-35.0] & 64.8 & 104.8 \\
CA15-3 (U/mL) [0.0-31.3] & 95.4 & 15.3 \\
SLX (U/mL) [0.0-38.0] & 330 & 46 \\
D-dimer (mg/mL) [0.0-1.0] & 6.2 & 3.2 \\
FDP (mg/mL) [0.0-4.9] & 8.5 & 6.2 \\
fibrinogen (mg/dL) [200-400] & 438 & 272 \\
\hline
\end{tabular}

TM tumor maker, CT coagulation test, POW4 postoperative week 4, CA19-9 carbohydrate antigen 19-9, CEA carcinoembryonic antigen, CA125 cancer antigen 125, CA15-3 Cancer antigen 15-3, SLX Sialyl Lewis X, FDP fibrin degradation product 

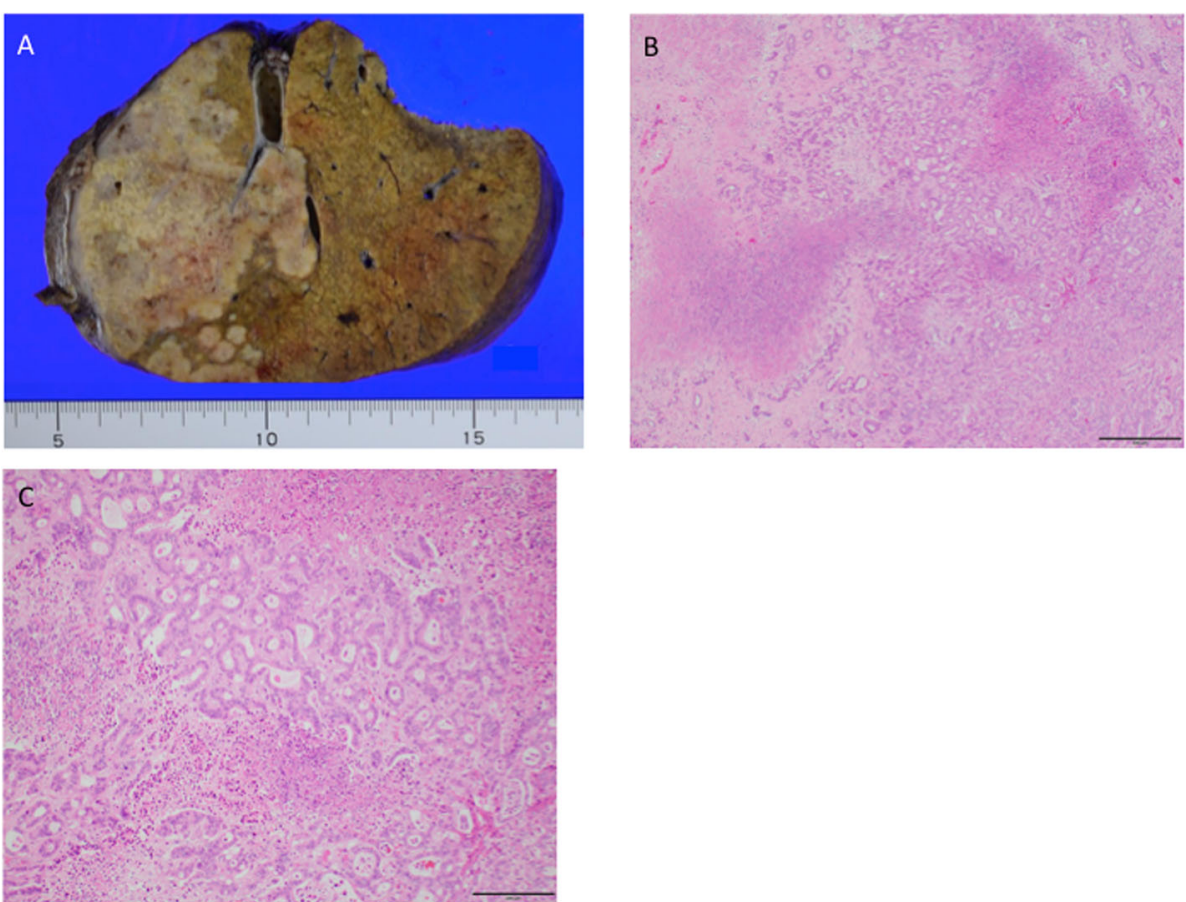

Fig. 2 Intrahepatic cholangiocarcinoma. a Cut surface of the formalin-fixed liver with solid masses in the right lobe. b Hematoxylin and eosin staining. Magnification, $\times$ 40. Scale bar, $500 \mu \mathrm{m}$. c Hematoxylin and eosin staining. Magnification, $\times 100$. Scale bar, $200 \mu \mathrm{m}$

A
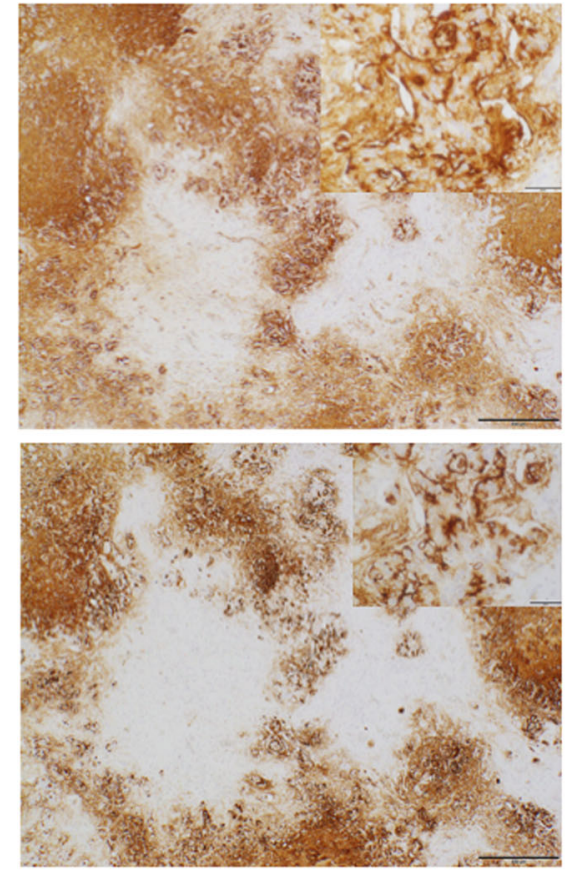
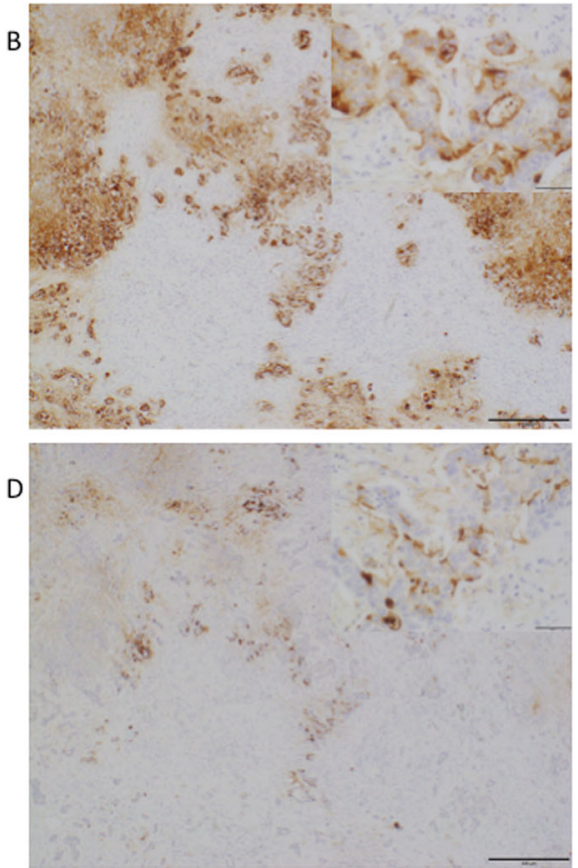

Fig. 3 Immunohistochemical findings. Immunohistochemistry for CA19-9 (a), CEA (b), MUC-1 (c), and CA125 (d). Magnification, $\times 40$ and $\times 200$. Note strong expression of CA19-9, CEA, and MUC-1 in the cytoplasm and membrane of cholangiocarcinoma cells. CA125 expression in the cytoplasm of cholangiocarcinoma cells is weak and partial. Scale bar $500 \mu \mathrm{m}$ and $50 \mu \mathrm{m}$. CA19-9, carbohydrate antigen 19-9; CEA, carcinoembryonic antigen; MUC-1, cell surface-associated mucin 1; CA125, cancer antigen 125 


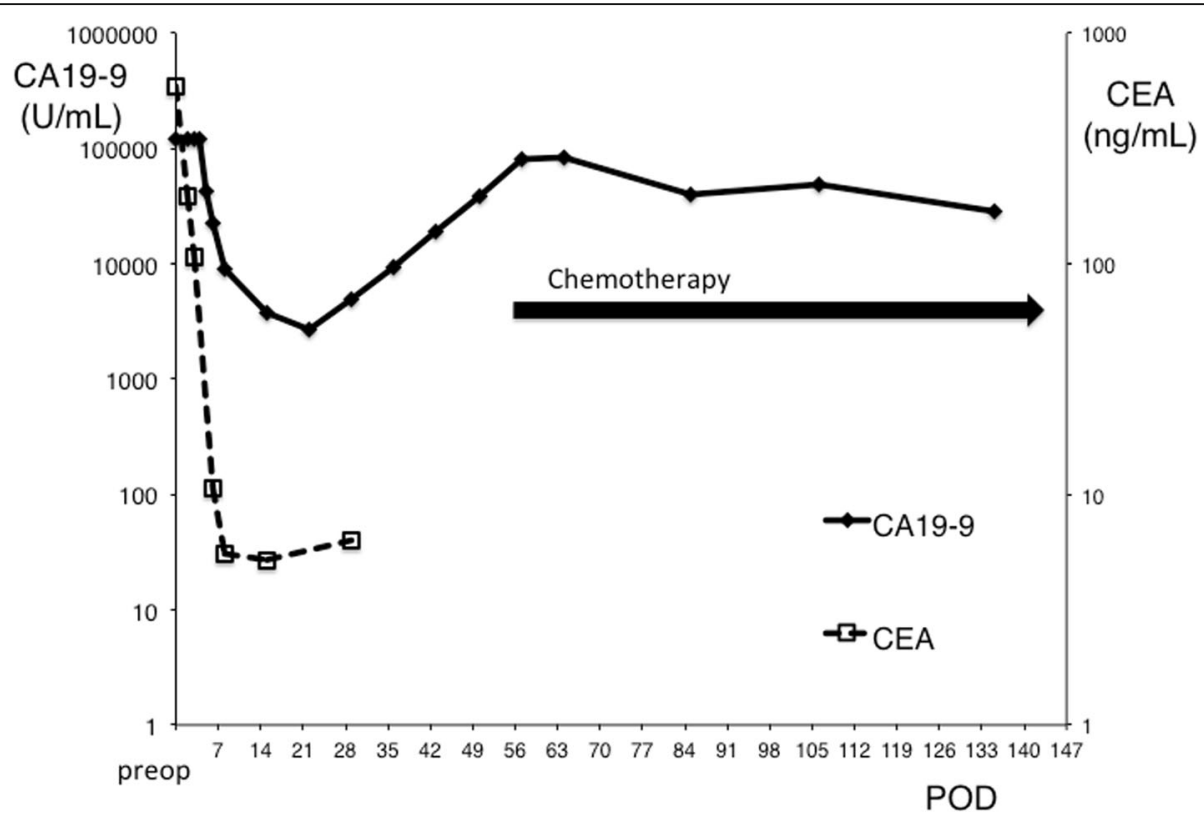

Fig. 4 Perioperative changes in CA19-9 and CEA levels. Carbohydrate antigen 19-9; CEA, carcinoembryonic antigen; POD, postoperative day

current patient underwent right hepatectomy and received systemic chemotherapy; she was successfully discharged and has been followed without further thrombosis during the 6 months after surgery.

Because most patients of Trousseau's syndrome and intrahepatic cholangiocarcinoma are at an advanced stage by the time of diagnosis, it is difficult for them to undergo curative treatment $[2,3,7,9]$. Therefore, the prognosis of the patients with Trousseau's syndrome and intrahepatic cholangiocarcinoma is very poor $[3,7$, $8]$. Because preoperative evaluation suggested that curative resection was possible in this case, we chose surgical resection for the treatment of the original tumor under the anti-coagulation prophylaxis, even though there was a possibility of further episodes of thrombosis. This strategy was fully explained to the patient and the family, and they completely agreed with our principle. In fact, Moustafa et al. recommended liver resection to
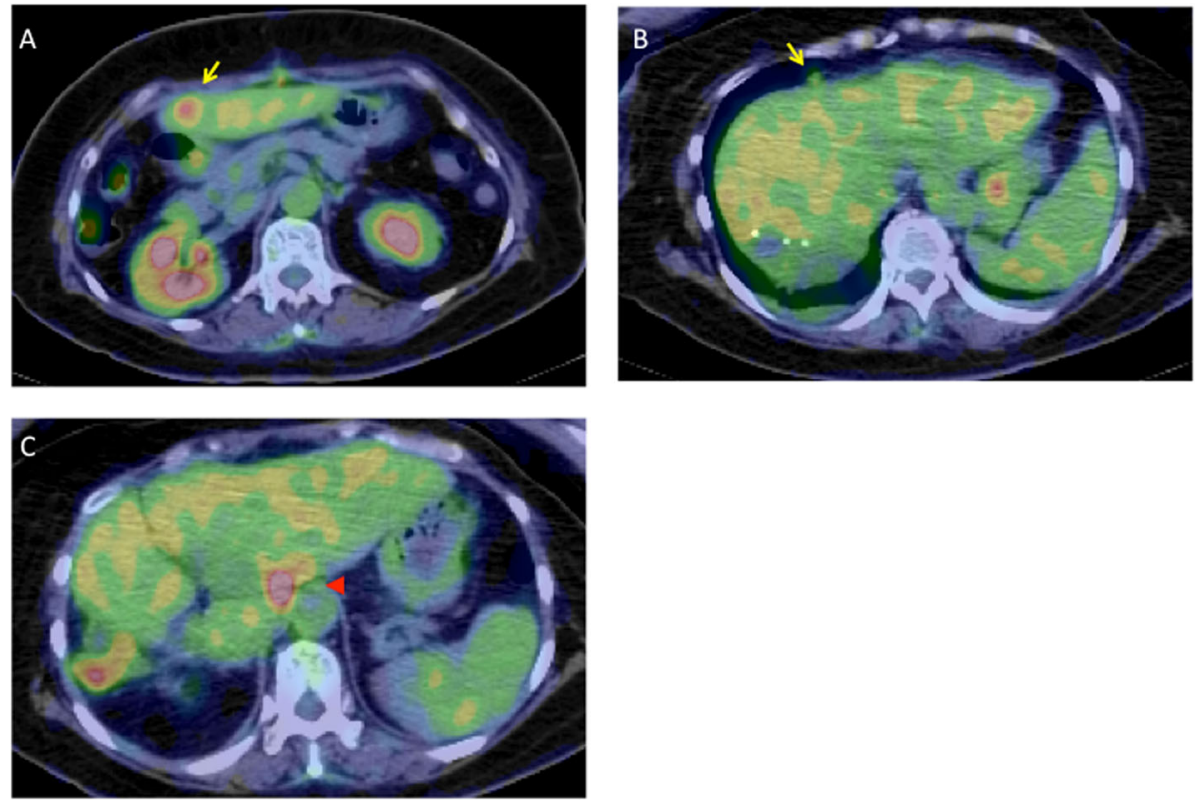

Fig. 5 Positron emission tomography (PET) findings on POW 7. a PET showing intrahepatic recurrence (arrow). b PET showing peritoneal dissemination (arrow). c PET showing hilar lymph node metastasis (arrowhead) 
intrahepatic cholangiocarinoma patients in stage III and IVa without multifocal lesions [10].

In the present case, the serum levels of CA19-9 were above the detectable range until 4 days after the surgery. The reported half-life of CA19-9 immediately after surgery is $0.5 \pm 0.1$ days [11]. Therefore, the preoperative serum CA19-9 level was estimated to be over 1,000,000 $\mathrm{U} / \mathrm{mL}$ in the present case. Given that tumor makers are usually considered only as diagnostic biomarkers, the pathological significance of an elevation in their levels is not seriously considered. However, the elevated tumor maker levels such as CA19-9, CEA, CA125, CA15-3, and Sialyl Lewis X (SLX) in the present patient might have been triggers for the observed thrombotic events (Table 1 ), as reported in a previous study [12]. Although thromboembolism in cancer patients is caused by multiple factors such as tissue factor, cytokines, and mucin, the tumor maker level should be kept as low as possible to avoid additional thrombotic events. Because Yuri et al. also reported a case of Trousseau's syndrome accompanied with cholangiocarinoma with high CA19-9 [8], our case enforced their speculation about the causative factors to Trousseau's syndrome. We should pay attention to thrombotic event in cholanginocarcinoma cases with high CA19-9. Although recurrence occurred just 7 weeks after the surgery in the present case, surgery might have been able to significantly reduce the CA19-9 levels. Immunohistochemistry showed an extremely strong expression of CA19-9. Even though the recurrence was not observed with conventional $\mathrm{CT}$, likely due to the small size of liver masses, her CA19-9 was elevated a few weeks after surgery. Her cholangiocarcinoma was considered to produce extremely high levels of CA19-9.

Heparin is widely preferred over oral anticoagulants to prevent thrombotic events in patients with Trousseau's syndrome [13]. In the present case, the infusion of unfractionated heparin was used as first-line treatment, and bridging anticoagulation with subcutaneous injection of low-molecular-weight heparin was performed during the perioperative period. Subsequently, the patient was switched to unfractionated heparin infusion followed by low-molecular-weight heparin in preparation for her discharge. The heparin treatment was successful, and the patient did not experience severe bleeding or thrombotic events in the perioperative period.

\section{Conclusion}

In conclusion, we herein reported the rare case of a patient with cholangiocarcinoma who presented with Trousseau's syndrome and successfully underwent right hepatectomy followed by systemic chemotherapy. As illustrated in the present case, an extremely high level of
CA19-9 might trigger Trousseau's syndrome in patients with cholangiocarcinoma.

\section{Abbreviations}

$\mathrm{CT}$ : Computed tomography; MRI: Magnetic resonance imaging; CA19-

9: Carbohydrate antigen 19-9; CEA: Carcinoembryonic antigen

\section{Acknowledgements}

The authors would like to thank Enago (www.enago.jp) for the English language review.

\section{Authors' contributions}

$\mathrm{RS}, \mathrm{YO}, \mathrm{SH}, \mathrm{SM}$, and $\mathrm{ON}$ were the attending physicians. KK performed the histological examination. YM, AT, HS, KS, KK, HH, ON, and YI participated in the design of the study. RS, YO, and $\mathrm{Yl}$ drafted the manuscript. The authors read and approved the final manuscript.

\section{Funding}

None of the authors received any funding for this study.

Availability of data and materials

Not applicable

Ethics approval and consent to participate

Not applicable

\section{Consent for publication}

Written informed consent was obtained from the patient. A copy of the written consent is available for review by the Editor-in-Chief of this journal.

\section{Competing interests}

The authors declare that they have no competing interests.

\section{Author details}

${ }^{1}$ Department of Surgery, Kumamoto Rosai Hospital, 1670 Takehara-machi, Yatsushiro, Kumamoto 866-8533, Japan. ²Department of Neurology, Kumamoto Rosai Hospital, 1670 Takehara-machi, Yatsushiro, Kumamoto 866-8533, Japan. ${ }^{3}$ Department of Diagnostic Pathology, Kumamoto Rosai Hospital, 1670 Takehara-machi, Yatsushiro, Kumamoto 866-8533, Japan.

Received: 25 February 2020 Accepted: 3 April 2020

Published online: 19 April 2020

\section{References}

1. Phlegmasia Alba Dolens TA. Lectures on clinical medicine, delivered at the Hotel-Dieu, Paris, vol. 5. London; 1865.

2. Varki A. Trousseau's syndrome: multiple definitions and multiple mechanisms. Blood. 2006;110:1723-9.

3. Blum MF, Ma VY, Betbadal AM, Bonomo RA, Raju RR, Packer CD. Trousseau's syndrome in cholangiocarcinoma: the risk of making the diagnosis. Clin Med Res. 2016;14:53-9.

4. Wahrenbrock M, Borsig L, Le D, Varki N, Varki A. Selectin-mucin interactions as a probable molecular explanation for the association of Trousseau syndrome with mucinous adenocarcinomas. J Clin Invest. 2003;112:853-62.

5. Soga Y, Taira K, Sugimoto A, Kurosawa M, Kira H, Su T, Doi K, Nakano A, Himura Y. Mitral valve nonbacterial thrombotic endocarditis: a rare multisurgery-tolerant survivor of Trousseau's syndrome. Surg Case Rep. 2018;4: 104.

6. Valle J, Wasan H, Palmer D, Cunninghamv D, Anthoney A, Maraveyas A, et al. Cisplatin plus gemcitabine versus gemcitabine for biliary tract cancer. N Engl J Med. 2010;362:1273-81.

7. Ching CK. Trousseau syndrome in a patient with cholangiocarcinoma. Am J Gastroenterol. 1991:86:928-9

8. Yuri T, Kato K, Hirohara Y, Kinoshita Y, Emoto Y, Yuki M, Yoshizawa K, Tsubura A. Trousseau's syndrome caused by intrahepatic cholangiocarcinoma: an autopsy case report and literature review. Case Rep Oncol. 2014;7:376-82.

9. Chan KM, Tsai CY, Yeh CN, Yeh TS, Lee WC, Jan YY, et al. Characterization of intrahepatic cholangiocarcinoma after curative resection: outcome, prognostic factor, and recurrence. BMC Gastroenterol. 2018;18:180. 
10. Moustafa M, Fasolo E, Bassi D, D'amico FE, Gringeri E, Pawlik TM, et al. The impact of liver resection on survival for locally advanced intrahepatic cholangiocarcinoma tumors: a propensity score analysis. Eur J Surg Oncol. 2020:46:632-7.

11. Yoshimasu T, Maebeya S, Suzuma T, Bessho T, Tanino H, Arimoto J, et al. Disappearance curves for tumor markers after resection of intrathoracic malignancies. Int J Biol Markers. 1999;14:99-105.

12. Awkar N, Amireh S, Rai S, Shaaban H, Guron G, Maroules M. Association between level of tumor markers and development of VTE in patients with pancreatic, colorectal and ovarian Ca: retrospective case-control study in two community hospitals. Pathol Oncol Res. 2018;24:283-7.

13. Ikushima S, Ono R, Fukuda K, Sakayori M, Awano N, Kondo K. Trousseau's syndrome: cancer-associated thrombosis. J Clin Oncol. 2016;46:204-8.

\section{Publisher's Note}

Springer Nature remains neutral with regard to jurisdictional claims in published maps and institutional affiliations.

\section{Submit your manuscript to a SpringerOpen ${ }^{\circ}$ journal and benefit from:}

- Convenient online submission

- Rigorous peer review

- Open access: articles freely available online

High visibility within the field

- Retaining the copyright to your article

Submit your next manuscript at $\boldsymbol{\nabla}$ springeropen.com 